\title{
The polar average reference effect: a bias in estimating the head surface integral in EEG recording
}

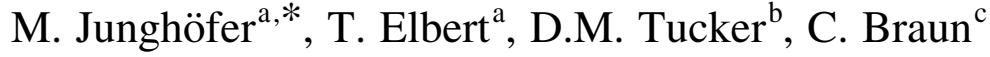 \\ ${ }^{a}$ Department of Psychology, University of Konstanz, Konstanz, Germany \\ ${ }^{\mathrm{b}}$ Department of Psychology, University of Oregon Electrical Geodesics Inc., Oregon, USA \\ ${ }^{\mathrm{c}}$ Department of Psychology, University of Tübingen, Tübingen, Germany
}

Accepted 8 February 1999

\begin{abstract}
A reference-independent measure of potential is helpful for studying the multichannel EEG. The potentials integrated over the surface of the body is a constant, i.e. inactive across time, regardless of the activity and distribution of brain electric sources. Therefore, the average reference, the mean of all recording channels at each time point, may be used to approximate an inactive reference. However, this approximation is valid only with accurate spatial sampling of the scalp fields. Accurate sampling requires a sufficient electrode density and full coverage of the head's surface. If electrodes are concentrated in one region of the surface, such as just on the scalp, then the average is biased toward that region. Differences from the average will then be smaller in the center of the region, e.g. the vertex, than at the periphery. In this paper, we illustrate how this polar average reference effect (PARE) may be created by both the inadequate density and the uneven distribution of EEG electrodes. The greater the coverage of the surface of the volume conductor, the more the average reference approaches the ideal inactive reference. (C) 1999 Elsevier Science Ireland Ltd. All rights reserved.
\end{abstract}

Keywords: EEG reference; EEG average reference; High resolution EEG; Evoked potentials; Signal to noise ratio; Standard deviation

\section{Introduction}

The electroencephalogram (EEG) is measured with a differential amplifier, such that the voltage time series reflects the difference in electrical potential between two electrodes. It is often assumed that one electrode site is an inactive reference, such that the potential at that site is constant across time. Yet this assumption has been known to be incorrect for many years (Nunez, 1981; Vaughan, 1982; Lehman and Skrandies, 1984). There is no site on the surface of the human head that can be assumed to remain at a constant potential during the activity of brain electrical sources of unknown locations and orientations (Nunez, 1981). Rather, the 'reference' sites such as the earlobes, mastoids, or nose, can be shown to vary in their electrical potentials during the event-related potential (ERP) epoch. These reference sites are influenced differentially by differing ERP components, such that the reference error varies unpredictably with experimental conditions that manipulate ERP components.

* Corresponding author. Tel.: + 49-7531-88-4539; fax: + 49-7531-882891.

E-mail address: markus junghoefer@uni-konstanz.de (M. Junghöfer)
Even for sites traditionally used as an 'inactive' reference, such as the earlobes, the variation of the potential over time must not be neglected. Not only may sources in the nearby temporal lobe affect this reference site, but also distant generators whose electrical fields propagate to the reference site through volume conduction.

The study of scalp topography, therefore, requires a reference-independent measure of the potential field. Some researchers have turned to the current density measure, estimated by the Laplacian derivation (the second spatial derivative) of the potential surface, which is referenceindependent. However, adequate spatial sampling is required to compute the spatial derivative, such that current density measures with 19- or 32-channel recordings will be unstable and inaccurate (Srinivasan et al., 1996; Junghöfer et al., 1997). Furthermore, even for high spatial density EEG, the second spatial derivative is much more noise vulnerable than the scalp potential distribution. Problems result from the inability to interpolate accurately (and thus to create accurate derivatives) with inadequate spatial sampling (below the spatial Nyquist frequency) and from the errors in determining the electrode positions. Furthermore, the current density measure is insensitive to deep sources, whereas the potential measure reflects deep and 

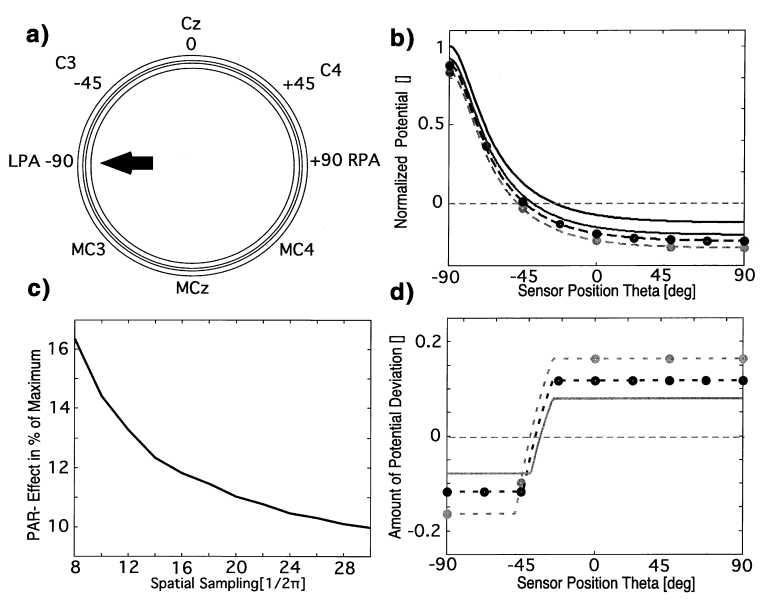

Fig. 1. (a) Illustration of the source and sensor configuration. Within a spherical volume model, consisting of 4 layers of homogeneous conductivity, a radial dipole was positioned a depth of $11 \mathrm{~mm}$ below the assumed cortical surface. Its direction was set to $-90^{\circ}$. (b) The black solid line refers to the potential distribution of the source indicated in Fig. 1a when referenced to the true average-reference. Distribution after transformation to an average reference based on the international 10-20 electrode configuration (gray dashed line, electrode positions are located at the 20\% distances and marked by circles), based on a high density electrode configuration (black dashed line, across the scalp: this density would correspond to a 129 electrode net) and based on a nearly continuous spatial sampling rate of $400 / 2 \pi$ (gray solid line). (c) Dependency of the maximum potential deviation $\left(-90^{\circ}\right)$ on the spatial sampling rate. (d) Variance of the absolute potential with transformation to average reference (style of lines correspond to the ones in Fig. 1b).

superficial sources. Because of these factors, a method is required to estimate the reference-independent potential.

An elegant solution to this problem might rely on the introduction of constraints based on physical principles. Specifically, the surface integral of the potential on a surface that completely encompasses all the active sources must be zero (Bertrand et al., 1985). The dipolar fields of each source are fully represented by the surface potentials. A positive radial source near the surface of the right side of the head produces a tight, high-amplitude positive field over that side. The opposing field is diffuse and low-amplitude over the left side, but with complete measurement the integrals of the two sides cancel exactly. The question for practical EEG recording is whether an average reference fulfils the criterion of adequate measurement of the surface integral.

Using simulated and real evoked potential data, Desmedt et al. (1990) and Tomberg et al. (1990) demonstrated that the average reference shifts across time, due to the lack of coverage of the head's surface. As pointed out by PascualMarqui and Lehmann (1993), dipole source modeling is not affected by the reference problem, as long as the potential fields are described properly (i.e. the location of the reference is included in the model). On the other hand, the reference bias, i.e. extent that the surface integral deviates from zero, can be an important clue to the extent of undersampling of the head surface potential field.
Furthermore, dipole modeling 'in the dark' can be dangerous: a careful study of scalp surface topography may be essential to eliminate unreasonable dipole solutions. Thus, an active reference may seriously affect various stages of the inspection, analyses and interpretation of EEG and ERP data (Dien, 1998). The least active reference is the one of choice, and with inadequate surface sampling, this is not necessarily the average reference. The present paper examines the activity of the average reference in dependence of spatial sampling and portion of scalp covered by electrodes, and uncovers the consequences.

As will be demonstrated, the first requirement to minimize the average reference problem is a dense spatial sampling. Current research EEG systems provide measurement with 64, 128, or 256 electrodes (Gevins et al., 1995; Tucker, 1993). The second requirement, for electrodes to cover the whole surface, is more difficult. In the present paper, using both simulated source configurations as well as real data, we show the bias created when the average reference is computed from less than the whole surface of the volume conductor. In a first step, simulated data are constructed from variable source configurations. Then the differences between the potential distribution (referred to the 'true' average-reference) and the voltage distribution (referred to the average reference based on the detected potential) are quantified. The results of these simulations confirm what has been observed in empirical studies of the average reference in EEG and ERP recordings (Curran et al., 1993; Chung et al., 1996): the potential amplitudes are smaller for electrodes located in the center of the electrode array than for those located on the edge. We refer to this bias as the polar average reference effect (PARE). Since the unknown reference bias for a single time point changes across samples - as the volume conducted sources affect the reference differently over time - PARE varies over time. We then demonstrate that, although it is reduced in magnitude, the PARE can be shown to remain in 128-channel ERP data sets. Finally, we suggest two possible ways to correct the PARE and discuss the consequences of the PARE for the interpretation of the distribution of scalp potentials and statistical analyses.

\section{Methods and results}

\subsection{Simulations of the PARE with absolute potential fields}

With simulations of a 4-sphere model of head conductivity, we can compute the surface integral and then measure the bias of the average reference (PARE) systematically. This section describes the calculations of various electrode and source configurations with respect to the PARE.

Any arbitrary source configuration can be described as the sum of a number of stationary current dipoles. Hence, it is sufficient to consider the effect of one such single current dipole. The head was modeled as an isotropic 

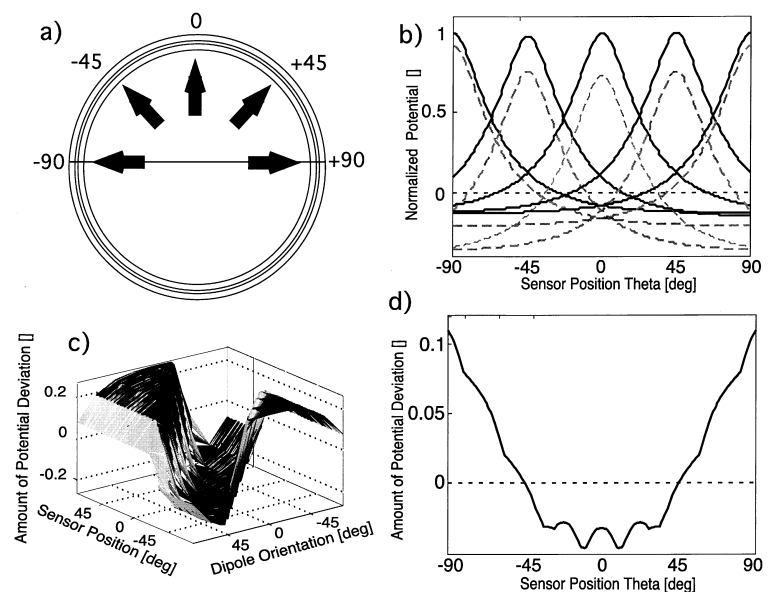

Fig. 2. (a) The radial dipole is placed at the same depth as the one in Fig.1, but its location is varied from -90 to +90 degrees. (b) Each black solid line indicates the potential distribution of one such source illustrated in Fig. 2a referenced to an imaginary zero-reference. Gray dashed lines show distributions after transformation to average reference based on a spatial sampling rate of $400 / 2 \pi$. (c) Magnitude of change of the absolute potential with transformation to average reference. The $x$ - and $z$-axes correspond to the axes in Fig. 1d. The $y$-axis illustrates the orientation of the assumed radial dipoles, varying between -90 and $90^{\circ}$. (d) Amount of deviation for the distributions given in Fig. $2 \mathrm{c}$ averaged across the different dipole locations as a function of sensor position.

volume conductor of spherical geometry composed of 4 homogeneous layers, each with a different conductivity (Cuffin and Cohen, 1979). The layers model scalp, skull, cerebra-spinal fluid (CSF), and brain with

$$
\begin{aligned}
\sigma_{\text {brain }} & =\sigma_{1}=(300 \Omega \mathrm{cm})^{-1}, \sigma_{\mathrm{CSF}}=\sigma_{2}=3 \sigma_{\text {brain }}, \sigma_{\text {skull }}=\sigma_{3} \\
& =\sigma_{\text {brain }} / 80, \sigma_{\text {scalp }}=\sigma_{4}=\sigma_{\text {brain }}
\end{aligned}
$$

The radius of the head $R$ was assumed to be $9.20 \mathrm{~cm}$, the radius of the brain $8.15 \mathrm{~cm}$, the thickness of skull and scalp were each set to $4.2 \mathrm{~mm}$, and the thickness of the CSF to 2.1 $\mathrm{mm}$. Due to the spherical symmetry, it is sufficient to vary the location of the sources in the two dimensional $r /$ theta (radius/polar angle) plane and to restrict the configuration to electrodes in the same plane. The following variables remain: position of the source and the orientation of its dipole moment, proportion of the sphere covered with electrodes and spatial sampling. Any possible orientation of the dipole moment can be constructed from the sum of its radially and its tangentially polar components (theta orientation). Fig. 1a illustrates an example of such a source and electrode configuration: the polar angle $\vartheta$ varies from left mastoid $\left(-90^{\circ}\right)$ via $\mathrm{C} 3\left(-45^{\circ}\right), \mathrm{Cz}\left(0^{\circ}\right), \mathrm{C} 4\left(+45^{\circ}\right.$ to the right mastoid $\left(+90^{\circ}\right)$. A radial dipole has an orientation of $90^{\circ}$, is positive and its depth (radius) is set at $16 \mathrm{~mm}$ below the cortical surface. In this and the following examples, the electrode configuration is symmetrical with respect to the vertex. The spatial sampling rate can be calculated using the corresponding positions located on the opposite side of the sphere. There are 8 electrodes on the circle $\left(360^{\circ}\right)$, resulting in a spatial sampling rate of $8 / 2 \pi$.

The black solid line in Fig.1b refers to the 'ideal' potential distribution (the 'true' average reference) of the dipole as described in Fig. 1a. The abscissa refers to the polar angle of the scalp positions. The proportion of coverage of the assumed electrode configuration corresponds to a half circle, i.e. $180^{\circ}$. All other lines in Fig. 1b show the potential distributions subtracted by an average reference potential which was computed using different spatial sampling rates. As is to be expected, the average reference effect becomes smaller as the spatial sampling rate increases. But even for a near continuous spatial sampling (gray solid line), a distinct effect remains due to the insufficient coverage of the surface of the sphere with electrodes only on the scalp. For a 30-channel recording (gray dashed line), the effect size amounts to approximately $16 \%$ of the maximal amplitude. This ratio drops to $11 \%$ for a 128-channel recording (black dashed line) and would remain $8 \%$ for continuous sampling (gray solid line), where the bias is due only to the insufficient coverage. As illustrated in Fig. $1 \mathrm{c}$, this effect size, thus decreases logarithmically with the spatial sampling rate. Fig. 1d shows the change of the potential that occurs when the average reference is subtracted.

The generalization of these effects to arbitrary radial source orientations of comparable depth is demonstrated in Fig. 2. Here we consider the locations and orientations that are exemplified by the course of the potential distribution prior to, and after, the transformation, displayed in Fig. $2 b$.

As is to be expected, the effect of transformation to average reference exhibits a cosinal distribution. Consequently, the effect is largest for a dipole pointing towards the vertex.
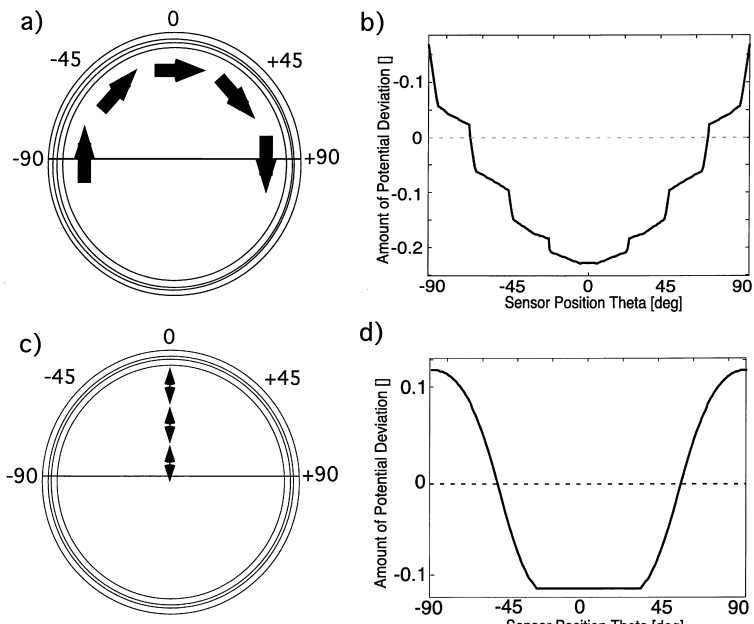

d)

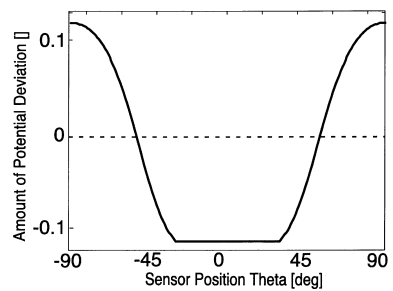

Fig. 3. (a) With retention of depth (like Figs. 1 and 2) a tangential dipole varies in location between -90 and +90 degrees. (b) as in Fig. 2d. (c) In this set of examples a radial dipole is placed at different depths $(11-70 \mathrm{~mm})$. Calculations are also performed for reversed polarities of the source. (d) as in Fig. 2d. 

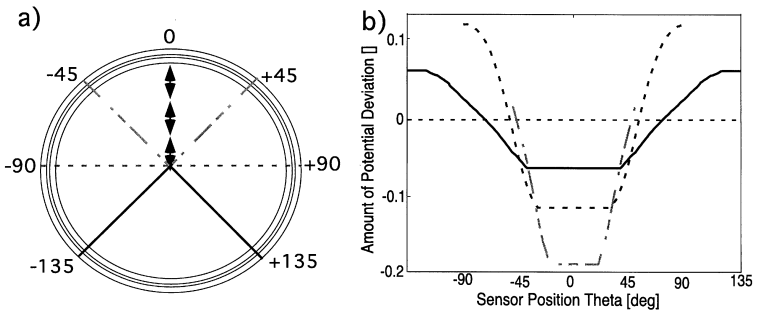

Fig. 4. Dependence of the PARE on the fraction of the head surface covered by electrodes. (a) The set of source configuration is the same as in Fig. 3b. The angle describing the fraction of the head surface covered by electrodes varies from 270 (solid line) over 180 (dashed line) to 90 degrees (dashed dotted line). (b) Mean value of the distributions calculated over all assumed source configurations, like illustrated in Fig 3c. Linestyle as in Fig. 4a. Means of the distributions over the source configurations assumed in Figs. 2 and $3 \mathrm{a}$ are not qualitatively different from the one in Fig. 3b. Therefore, these results are not shown.

Fig. 2c corresponds to Fig. 1d; it describes the generalized effect of change in absolute value. While the abscissa (sensor position) is the same as in Fig. 1d, the ordinate represents the dipole orientations indicated by Fig. 2a. The last row of the matrix illustrated in Fig. 2c (last slice of the figure, dipole orientations $=-90^{\circ}$ ) is identical to Fig. $1 \mathrm{~d}$. The average across all possible source orientations is displayed in Fig. $2 \mathrm{~d}$ and shows a u-shaped polar distribution in the amount of potential deviation. Potentials from inferiorly located sensors are enlarged in amplitude while potentials from superior sensors appear smaller than they really are.

Let us consider this PARE more closely. The change to tangentially oriented dipoles (in Fig. 3a) results in a qualitatively similar distribution (Fig. 3b). Inversion of the polarity will, of course, not alter this distribution. The order of the size of the PARE is considerably larger for inferiorly located tangential sources than for the radial ones, since the tangential sources will give rise to greater potentials in those areas of the head surface that are not covered by the sensor array. The polarization of the supratemporal plane resulting from auditory stimulation is an example of a source distribution extending beyond the typical recording array.

When, in the next step, the average reference effect is calculated for different depths of the dipole locations and different polarities (Fig. 3c), the polar bias again appears (Fig. 3d). Hence, considering the effect across all possible source configurations, the size of the potential will be magnified at sensors in inferior regions, but diminished at the superior locations. Any data set that is transformed to average reference is contaminated by the PARE. The magnitude of this effect, however, gets smaller with increasing proportion of area covered by the sensory array (Fig. 4) and, also, with increasing spatial sampling (Fig. 1c).

The consequences of the PARE may be illustrated for a 3 dimensional arrangement of sources and sensors. In this
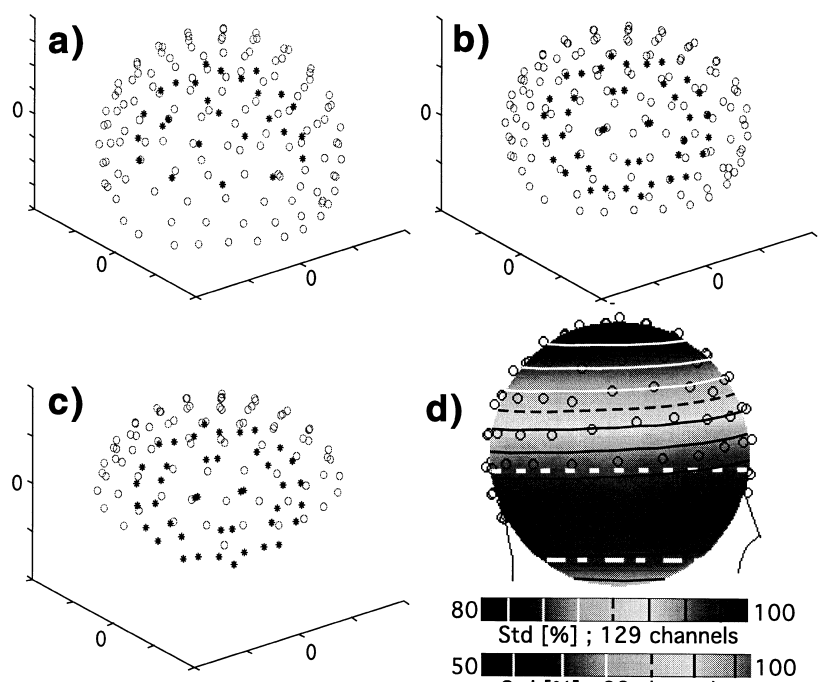

d)
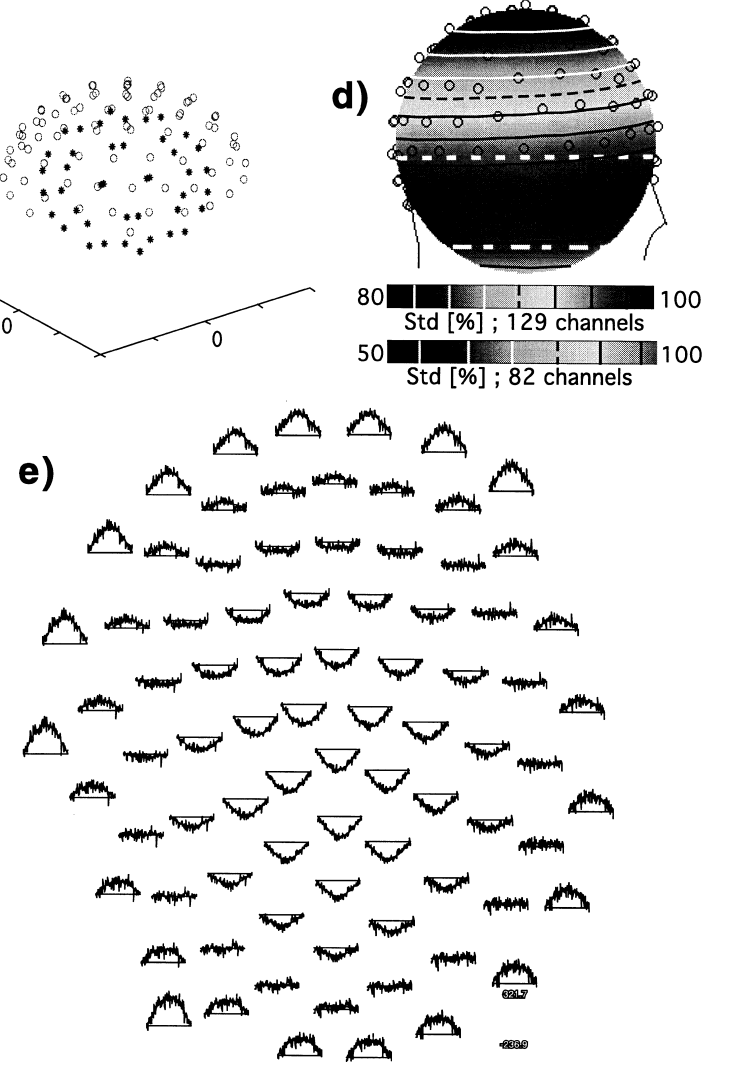

Fig. 5. (a-c) Different source (stars) and sensor configurations (circles). Stationary dipoles with time-varying orientation but fixed in dipole moment were assumed at these source locations in order to simulate correlated noise (see text for further details). (d) Distribution of the standard deviation calculated over 100 different dipole orientation configurations using source locations as given in (a) (upper color axes) or (c) (lower color axes). Source configuration as in (a). (e) A radial dipole is placed underneath the vertex (marked by an $\mathrm{x}$ ) and activated with a sinusoidal half wave. The potential generated by this dipole is averaged across 100 epochs whereby randomized 'noise' sources were assumed over time and epochs. The mean amplitude of the potential distribution generated by these random sources was set at 4 times the mean amplitude of the potential distribution generated by the 'signal source', i.e. the mean signal to noise ratio was set to 1:4. Sensor and source configuration correspond to the ones illustrated in Fig. 5c. After averaging across the 100 trials, one might expect a signal to noise ratio of 10:4 at any given sensor. This, however, is not the case. Due to the PAR effect, the signal to noise ratio varies from 10:4 at the vertex electrode to $5: 4$ at the maximally inferior located electrode.

example, we simulate spatially correlated noise, such as is created by the background EEG in an averaged ERP study. We assume dipoles are located at 42 different locations, the amplitude of which are constant across a hundred trials while the direction in space of the moment varies randomly. 


\section{Left Index Finger}

a)

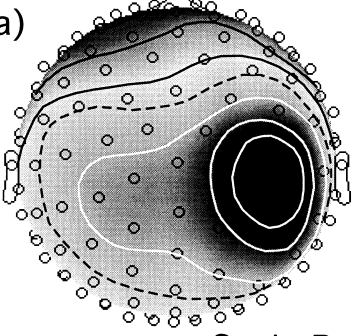

Scalp
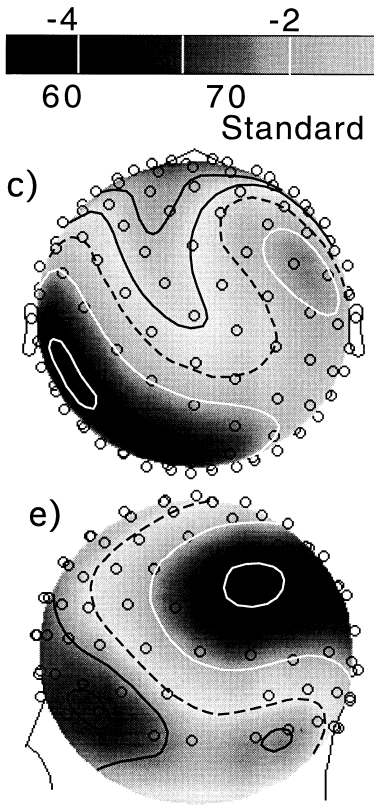

Right Index Finger

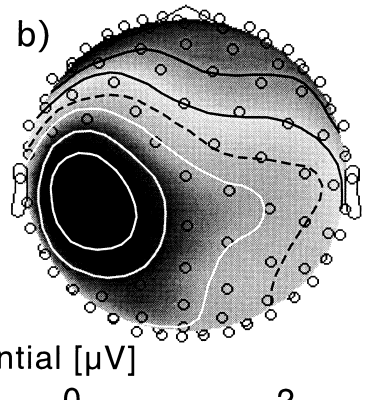

b)

2

\begin{tabular}{l|cc}
\hline & & \\
80 & 90 & 100
\end{tabular}

eviation [\%]
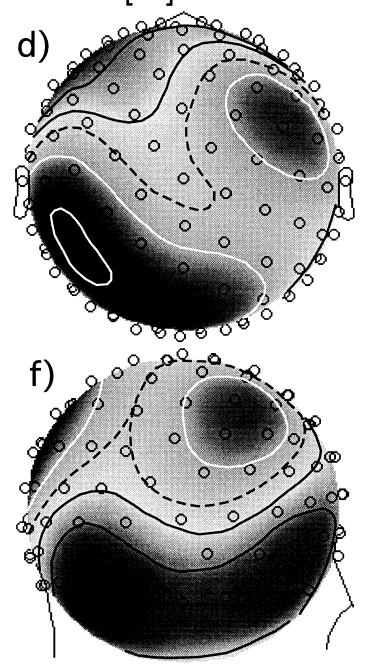

Fig. 6. (a,b) Illustrate the potential distribution of a Bereitschaftspotential recorded during a standard paradigm. The subject was asked to voluntarily press a button with the left (left row) or right (right row) index finger and did so about once every $3 \mathrm{~s}$. The potentials were averaged across an interval from $500 \mathrm{~ms}$ prior to the onset of the motoric response. As is to be expected, negativity becomes maximal over the contralateral motor cortex. The color coding corresponds to the upper color scale. (c-f) Distribution of the standard deviations averaged across the same time segment as in $(a, b)$ for the left finger with top view (c) and left view (e) and for the right finger with top view (d) and right view (f). Color coding corresponds to the lower color scale. The standard deviation distribution looks surprisingly similar regarding the distinct lateralization of the potential distributions (a) and (b). See text for further details.

More precisely, the dipoles were located at the corners of an icosohedron (Bucky ball) having a radius of $6.5 \mathrm{~cm}$. A typical 128-channel whole-head electrode configuration was assumed.

The active dipoles in Fig. 5a were restricted to those 26 spatial locations that have positive $\mathrm{z}$ values. Hence, the area covered by the modeled 128-channel recording was considerable. Fig. 5d presents the interpolated distribution of standard deviations across all trials, after transformation of the simulated potentials to average reference. Despite the considerable coverage by the sensors, a strong PAR effect remains; the standard deviation of the most superiorly located electrode, $\mathrm{Cz}$, amounts to only $80 \%$ of the most inferior ones. If all 42 dipoles are activated, the result is qualitatively the same, but the difference in standard deviation increases by another 5\%. The reason for this lies in a secondary AR effect; active sources that are located below the area covered by the sensors affect the inferiorly located sensors to a greater extent than the ones located at superior positions. In the present example, the secondary effect is relatively small because the electrode configuration, as displayed in Fig. 5b, has considerable coverage - even for the globally distributed source configuration. Even then, there are only a few model dipoles that are located beyond the covered space.

This effect, however, will gain increasing weight if coverage is insufficient. In Fig. 5c only the 82 electrode positions with a positive $z$ value were used in the calculation of the average reference; these positions were located above the upper dashed line of Fig. 5d. The polar distribution is qualitatively similar, but larger; the standard deviation at the vertex electrode drops to only $50 \%$ of that for the most inferiorly located sensors. This area of coverage is by no means unrealistic; it reflects that of the international 10-20 system. To make this point even more explicit, the effect can be illustrated for a simple focal active source that varies as a function of time. Assuming the 82-sensor configuration and 42 source locations, as in Fig. 5c, in Fig. 5e, an additional radial source, located below the vertex, was consistently activated in all the trials with a sinusoidal time course and an amplitude that amounted to $25 \%$ of the total amplitude averaged across all 42 noise sources. The signal-to-noise ratio was 1:4 in one trial and, hence, 2.5 after averaging across the 100 trials. The potential distribution shows a pronounced PAR effect (Fig. 5e) with a signal-to-noise ratio of only 1.25 at the inferior locations as opposed to 2.5 at the vertex electrode. This dependence of the signal-to-noise ratio on the sensor location must not be neglected.

\subsection{Examples of the PARE in dense array ERP recordings}

Of course, because the differences in potential can be reattributed within a common reference recording array through subtraction, the PARE is independent of the choice of reference electrode for recording purposes. Although the PARE decreases with the improved spatial sampling of a dense array EEG recording, the following example suggests that it can still be observed.

The upper column of Fig.6 (a,b) illustrates the distribution of a Bereitschaftspotential recorded in a standard design: the subject was asked to voluntarily press a button with the left (left column in Fig. 6), or right (right column), index finger about every $3 \mathrm{~s}$. Data was low-pass filtered with $40 \mathrm{~Hz}$ and backward averaged, using the EMG onset as a trigger. Then the mean was calculated for each sensor across an epoch beginning $500 \mathrm{~ms}$ prior to the motor response. The Bereitschaftspotential shows the typical frontocentral distribution with contralateral dominance. 


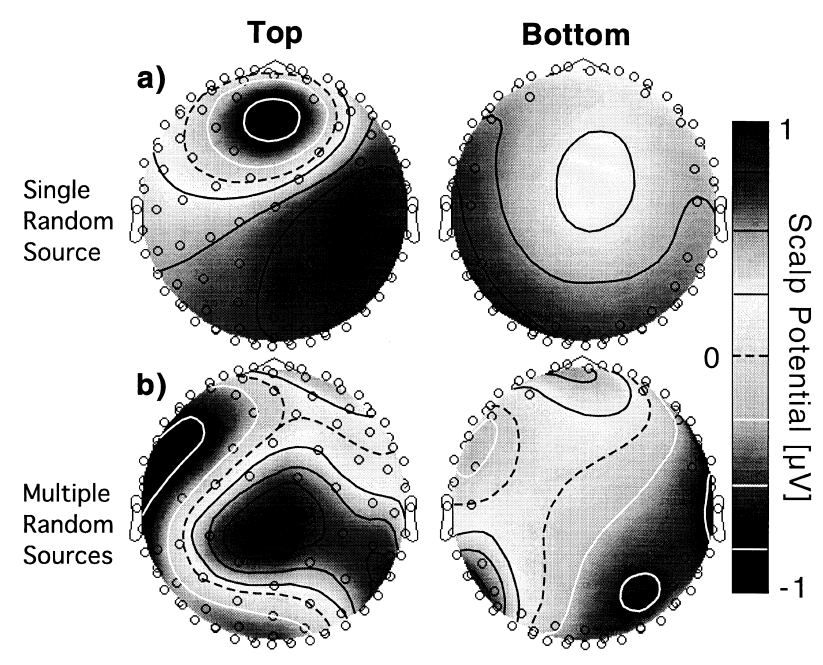

Fig. 7. Illustration of activity that is measured (left column) and that goes undetected (right column), even in the 129 sensor net for (a) a single dipole and (b) a multiple random source configuration. In both cases, this activity is different from zero, giving rise to a PARE. The information from the 129 electrodes may be used to estimate the potential on the surface not covered by electrodes using a spherical spline interpolation.

The lower columns of Fig. 6c-f illustrate the scalp distribution of the standard deviations, averaged over the time interval mentioned above. These standard deviations of the averaged ERP primarily reflect the background (responseasynchronous) EEG of the recording.

The standard deviation distributions for left and right movement look surprisingly similar, although the lateralization of the potential distributions differ distinctly. In both cases, the standard deviation is smallest in left parietal regions. Higher standard deviations are detectable in frontal and right occipital regions.

It is tempting to conclude that the generators in the sensory motor cortex produce a rather constant activation across trials while other areas vary greatly from trial to trial in their degree of activation. Unfortunately, these very interesting distributions are contaminated by the PAR effect. As outlined above, we can not reliably estimate the magnitude of the PARE unless the generator structure is known. Therefore, any interpretation of the distribution of standard deviation calls for considerable caution.

\subsection{PARE correction}

As obvious from the theoretical considerations, the PAR effect is a consequence of a failure to fully sample the surface potential on the head. More precisely, it is the average potential across the area not covered by electrodes, which is not measured. If it could be estimated, the PAR effect might be compensated for. The following section examines this possibility. For this purpose, we again assume - as in Fig. 5 - 42 source locations. The source dipoles vary randomly in direction and amplitude of their moments across 200 trials. Let us begin assuming one active source per trial.

If there is no measurement error, the surface integral, i.e. the average potential across the head's surface, must be constant across time. Consequently, the average reference that results from averaging a potential across all electrodes using any reference electrode must also be constant, provided the sensors cover the head surface completely. The average potential for a 129-electrode recording which does not completely cover the body surface varies with brain activity. Across the 200-source configurations, its standard deviation amounts to about $20 \%$ of the standard deviation of the potential at a single electrode. (Note that for this simulation study, the standard deviations at the individual electrode sites are almost identical).

The standard deviation of the average divided by that of one of the individual electrodes can be described as the 'average reference coefficient'. The examples of the potential distribution presented in Fig. 7 depict the amount of activity missed by the 129 sensor net for a single dipole (Fig. 7a) and a multiple random source configuration (Fig. 7b). In both cases, this activity is different from zero, causing the PARE. The information from the 129 electrodes may be used to estimate the potential on the surface not covered by electrodes using a spherical spline interpolation. In a second step, the average reference can be computed for the entire surface using the results from the interpolation. This procedure reduces the standard deviation of the average reference to only $10 \%$ of the standard deviation across source configurations at any single electrode, i.e. it halves the error in estimating the average reference and, thus, also reduces the PARE.

The possibility of estimating the true average reference is facilitated if the sources are deep and the spatial sampling is high. Fig. 8 illustrates the dependency on both, the number of channels and the depths of source generators, to properly
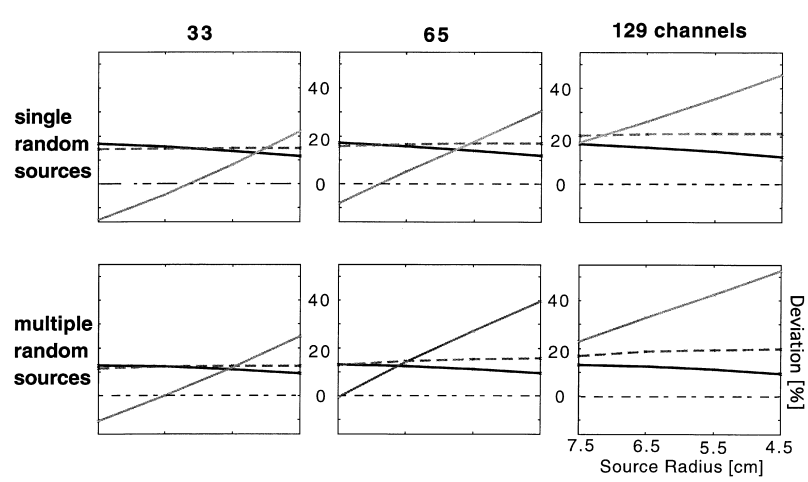

Fig. 8 . The possibility of estimating the true average potential is facilitated if the sources are deep and the spatial sampling is high. The diagrams illustrate the dependency on both, the number of channels and the depths of source generators, to properly estimate the average reference. The dashed gray lines indicate the uncorrected average reference coefficients, while the solid black lines indicate the corrected ones. The measure $\mu$ (gray solid lines) for the usefulness of the correction can be based on the average reference coefficient: $\mu=\left(1-\right.$ RestStd $_{\text {corr }} /$ RestStd $\left._{\text {uncorr }}\right) \times 100$ 
estimate the average reference. Again, a measure $\mu$ for the usefulness of the correction can be based on the average reference coefficient

$\mu=\left(1-\frac{\text { RestStd }_{\text {corr }}}{\operatorname{RestStd}_{\text {uncorr }}}\right) \times 100$

Generally, the average reference coefficient varies between 16 and 20\% (gray dashed lines). Since the proportion of the area covered by the sensor array increases with spatial sampling, the uncorrected average reference coefficient is a few percent greater for the 129-channel recording than for the 33 channel net. As the probability of detecting peaks in the potential distribution increases with the density of the array, the likelihood that the average reference is different from zero will also increase. Consequently, the PARE is likely to be greater for higher numbers of sensors given the same proportion of area covered. For the uncorrected average reference coefficient, the depth of the sources has little influence.

The measure $\mu$ for the usefulness of the correction increases nearly linearly with the number of sensors. With an average over single and multiple random sources of different depths we obtain $\mu=0 \%$ for the 33-channel configuration, meaning that PARE correction is not possible. Using 65 channels the averaged $\mu$ became $15 \%$ and in the case of a 129 -channel system the value doubles to $30 \%$. The implication is that the PARE is more accurately characterized when the full field topography is more accurately estimated with the 128-channel sampling.

Instead of using the spherical spline interpolation, an alternative approach can be based on the estimation of the source configuration and, subsequently, the calculation of the average potential from the forward solution of the estimated sources. Additional gain from this approach is limited.

\section{Discussion}

The inaccuracy of the average reference, described by the polar average reference effect, is substantial when the number of electrodes is small and when a small proportion of the surface of the head is covered. With increasing coverage and a high electrode density, the PARE is reduced. However, even if the surface of the head is covered by an electrode net extending across $270^{\circ}$, this effect is not negligible. It affects further steps of EEG analysis.

We emphasize again, that the problems arising from the PARE cannot be resolved by simply choosing a different type of reference. The only solution to minimizing the effect is to use a high density electrode array that covers as large a portion of the head surface as possible. Since current source density calculations are unstable and inaccurate when based on insufficient spatial sampling recordings (e.g. with 19- or 32-channel), these transformations for solving the reference dependencies should not be used with a small number of electrodes. We are currently evaluating to what extent an electrode that is inserted into the oral cavity might supplement a 128-channel geodesic sensor net to improve the characterization of the electrical fields at the inferior surface of the brain-case.

\section{Acknowledgements}

This study was supported by the Deutsche Forschungsgemeinschaft. The authors would like to thank Ursula Lommen and Irina Tuderoiu for collecting the experimental data.

\section{References}

Bertrand O, Perrin F, Pernier J. A theoretical justification of the average reference in topographic evoked potential studies. Electroenceph clin Neurophysiol 1985;62:462-464.

Chung G, Tucker D, West P, Potts G, Liotti M, Luu P, Hartry A. Emotional expectancy: brain electrical activity associated with an emotional bias in interpreting life events. Psychophysiology 1996;33:218-233.

Cuffin B, Cohen D. Comparison of the magnetoencephalogram and electroencephalogram. Electroenceph clin Neurophysiol 1979;47:132-146.

Curran T, Tucker D, Kutas M, Posner M. Topography of the N400: brain electrical activity reflecting semantic expectation. Electroenceph clin Neurophysiol 1993;88:188-209.

Desmedt J, Chaklin V, Tomberg C. Emulation of somatosensory evoked potential (SEP) components with the 3-shell head model and the problem of 'ghost potential fields' when using an average reference in brain mapping. Electroenceph clin Neurophysiol 1990;77:243-258.

Dien J. Issues in the application of the average reference: review, critiques, and recommendations, Behav Res Methods Instrum Comp 1998;30:3443.

Gevins A, Leong H, Smith M, Le J, Du R. Mapping cognitive brain function with modern high-resolution electroencephalography. Trends Neurosci 1995;18(10):429-436.

Junghöfer M, Elbert T, Leiderer P, Berg P, Rockstroh B. Mapping EEGpotentials on the surface of the brain: a strategy for uncovering cortical sources. Brain Topog 1997;9(3):203-217.

Lehman D, Skrandies W. Spatial analysis of evoked potentials in man - a review. Progress in Neurobiology 1984;23:227-250.

Nunez, P., Electric Fields of the Brain: The Neurophysics of EEG. New York: Oxford University Press, 1981.

Pascual-Marqui R, Lehmann D, et al. Topographic maps, source localization inference, and the reference electrode: comments on the paper by Desmedt .Electroenceph clin Neurophysiol 1993;88:532-533.

Srinivasan R, Nunez P, Tucker D, Silberstein R, Cadusch P. Spatial sampling and filtering of EEG with Spline-Laplacians to estimate cortical potentials. Brain Topog 1996;8:355-366.

Tomberg C, Noel P, Ozaki I, Desmedt J. Inadequacy of the average reference for the topographic mapping of focal enhancements of brain potentials. Electroenceph clin Neurophysiol 1990;77:259-265.

Tucker D. Spatial sampling of head electrical fields: the geodesic sensor net. Electroenceph clin Neurophysiol 1993;87:154-163.

Vaughan HG. The neural origins of human event-related potentials. Ann N Y Acad Sci 1982;338:125-138. 